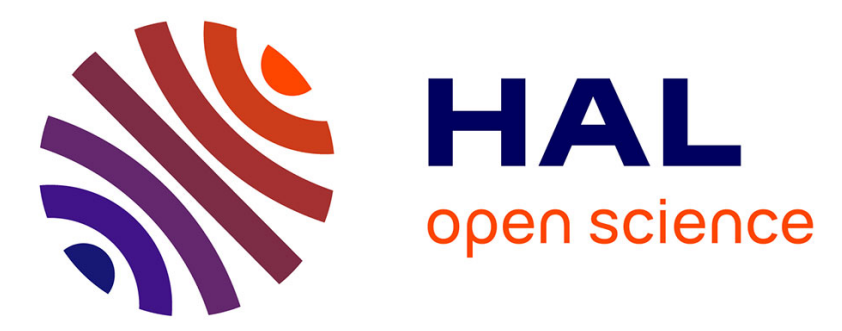

\title{
The Condorcet Efficiency of the Preference Approval Voting and the Probability of Selecting the Condorcet Loser
}

Eric Kamwa

\section{To cite this version:}

Eric Kamwa. The Condorcet Efficiency of the Preference Approval Voting and the Probability of Selecting the Condorcet Loser. Theory and Decision, 2019, 87 (3), pp.299-320. hal-01786121v2

\section{HAL Id: hal-01786121 \\ https://hal.univ-antilles.fr/hal-01786121v2}

Submitted on 29 Nov 2018

HAL is a multi-disciplinary open access archive for the deposit and dissemination of scientific research documents, whether they are published or not. The documents may come from teaching and research institutions in France or abroad, or from public or private research centers.
L'archive ouverte pluridisciplinaire HAL, est destinée au dépôt et à la diffusion de documents scientifiques de niveau recherche, publiés ou non, émanant des établissements d'enseignement et de recherche français ou étrangers, des laboratoires publics ou privés. 
myjournal manuscript No.

(will be inserted by the editor)

\title{
On the Condorcet Efficiency of the Preference Approval Voting and the Probability of Selecting the Condorcet Loser
}

\author{
Eric Kamwa
}

Received: date / Accepted: date

\begin{abstract}
Under Approval Voting (AV), each voter just distinguishes the candidates he approves of from those appearing as unacceptable. The Preference Approval Voting (PAV) is a hybrid version of the approval voting first introduced by Brams and Sanver (2009). Under PAV, each voter ranks all the candidates and then indicates the ones he approves. In this paper, we provide analytical representations for the probability that PAV elects the Condorcet winner when she exists in three-candidate elections with large electorates. We also provide analytical representations for the probability that PAV elects the Condorcet loser. We perform our analysis by assuming the assumption of the Extended Impartial Culture. This analysis allows us to measure at which extend, PAV performs better than AV both on the propensity of electing the Condorcet loser and on that of the non-election of the Condorcet loser.
\end{abstract}

Keywords Approval Voting · Ranking · Condorcet · Extended Impartial Culture · Probability

E. Kamwa $(\bowtie)$

LC2S UMR CNRS 8053, Université des Antilles, Faculté de Droit et d'Economie de la Martinique

E-mail: eric.kamwa@univ-antilles.fr 


\section{Introduction}

Popularized by Brams and Fishburn (1978), the Approval Voting (AV) rule is a voting system under which each voter approves (any number of) candidates that he considers as acceptable and the winner is the most-approved candidate. This rule has made (and continues to be) the subject of numerous research works in political science, economics and computer science. To have a quick overview of these works, the reader may refer to the books of Brams (2008), Brams and Fishburn (2007) and to the Handbook of Approval Voting edited by Laslier and Sanver (2010). Under AV, there is no need to rank the candidates as under the scoring rules ${ }^{1}$. This absence of rankings gave rise to a controversy between Saari and van Newenhizen (1988a,b) and Brams et al. (1988a,b). Saari and van Newenhizen (1988b) blame AV of hiding the real preferences of the voters which can be strict between the candidates approved by a voter. Brams and Sanver (2009) may have brought what appears as a possible response to this criticism by introducing the Preference Approval Voting (PAV). Under PAV, each voter ranks all the candidates then indicates the ones he approves. ${ }^{2}$ According to Brams and Sanver (2009), the winner under PAV is determined by two rules:

Rule 1: The PAV winner is the AV winner if ${ }^{3}$

i. no candidate receives a majority of approval votes (i.e approved by more than half of the electorate)

ii. exactly one candidate receives a majority of approval votes.

Rule 2: In the case that two or more candidates receive a majority of approval votes,

i. The PAV winner is the ones among these candidates who is preferred by a majority to every other majority-approved candidate.

ii. In the case of a cycle among the majority-approved candidates, then the AV winner among them is the PAV winner.

Brams and Sanver (2009) noticed that it is Rule 2 that clearly differentiate PAV from AV. They pointed out that for some situations where a Condorcet winner exists, this candidate may not be a PAV winner under each of the subcases of Rule 1 and Rule 2. When she exists, a Condorcet winner is a candidate who defeats each of the other candidates in pairwise comparisons. We know that AV always elects the Condorcet winner when she exists given that voters' preferences are dichotomous (Ju, 2010, Xu, 2010). This is no more the case when the voters' true preferences are assumed to be strict orderings (Gehrlein and Lepelley, 1998) or when indifference are allowed in the voters' true preferences (Diss et al., 2010). For large electorates, Gehrlein and Lepelley (1998) found that $\mathrm{AV}$ has the same Condorcet efficiency (probability of electing the Condorcet winner when she exists) as both the Plurality rule and the Antiplurality rule. ${ }^{4}$ Going from a more general framework, Diss et al. (2010) found that for large electorates and three candidates, AV performs better that both the Plurality rule and the Antiplurality rule on the Condorcet efficiency; they also found some scenarios under which AV performs better than the Borda rule. Their results were strongly reinforced by Gehrlein and Lepelley (2015).

As just stated, many works have been focused on the Condorcet efficiency of AV while nothing is known about PAV as no work has been done on the subject. Thus, one of objective of this paper is to try to fill this void for voting situations with three candidates by computing the Condorcet efficiency of PAV when indifference are allowed as in Diss et al. (2010). So, we provide a representation of the limiting probability of the Condorcet efficiency of PAV. All the computations are done under the extended impartial culture assumption introduced by Diss et al. (2010); this

\footnotetext{
1 A scoring rule is a voting rule under which voters give points to candidates according to the ranks they have in voter's preferences. The winner is the candidate with the highest total number of points.

2 Brams and Sanver (2009) also introduced the Fallback Voting under which voters only rank the candidates they approve. In this paper, we are not concerned with this rule.

${ }^{3}$ Here, we have chose to split Rule 1 into two. This will be helpful for our analysis.

4 The Plurality rule is a scoring rule under which each voter votes only for (gives one point to) his top ranked candidate and the winner is the ones with the highest total number first places; under the Antiplurality rule, the winner is the candidate with the fewest total number of last places.
} 
assumption will be defined later. By definition, it is obvious that PAV performs better than AV on electing the Condorcet winner when she exists. It would be interesting to measure the extent of this dominance. In order to better reflect this, we first provide a more general representation of the limiting probability of the Condorcet efficiency of AV. Then, the representation provided by Diss et al. (2010) comes as a particular case of ours.

Some works have looked on the probability that AV elects the Condorcet loser when she exists. A Condorcet loser is a candidate, when she exists, who is defeated by each of the other candidates in pairwise comparisons. Gehrlein and Lepelley (1998) showed that with more than three candidates and under the impartial culture assumption, AV is more likely to elect the Condorcet loser than the Plurality rule. For three-candidate elections, they showed that AV has the same probability of electing the Condorcet loser as both the Plurality rule and the Antiplurality rule. This result is a bit challenged by a recent paper by Gehrlein et al. (2016). Using impartial anonymous culturelike assumptions ${ }^{5}$ and considering a range of scenarios, Gehrlein et al. (2016) concluded that in three-candidate elections, AV is less likely to elect the Condorcet loser than both the Plurality rule and the Antiplurality rule. By definition of PAV, it comes that it is less likely to elect the Condorcet loser when she exists. The second objective of this paper is to focus on the probability that PAV elects the Condorcet loser when she exists. We provide for AV and for PAV, analytical representations of the limiting probability of electing the Condorcet loser when she exists under the extended impartial culture assumption. By doing so, we will highlight the extent of dominance of PAV on AV

The rest of the paper is structured as follows: Section 2 is devoted to basic notations and definitions. Section 3 presents our results on the Condorcet efficiency. Section 4 deals with our results on the probability of electing the Condorcet loser. Section 5 concludes.

\section{Preliminaries}

\subsection{Preferences in three-candidate elections}

Let $N$ be a set of $n$ voters $(n \geq 2)$ and $A=\{a, b, c\}$ a set of three candidates. We assume that voters rank all the candidates, indifference is allowed and they indicate which candidates they approve by underlining the names of the candidates ${ }^{6}$. So, there are 19 possible types of preferences. Following Diss et al. (2010), these 19 types of preferences can be partitioned into five classes of preferences:

- Class I: this class is made of voters with strict rankings and who only approve their top ranked candidates. These voters are labelled 1 to 6 in Table 1.

- Class II: voters in this class also have strict ranking and they approve their top two ranked candidates. These types of voters are labelled 7 to 12 in Table 1.

- Class III: in this class, voters are indifferent between their two preferred candidates or do not consider the difference significant enough to reveal their true strict preference. These types of voters are labelled 13 to 15 in Table 1.

- Class IV: Voters rank one candidate strictly above the two other between whom they are indifferent. These types of voters are labelled 16 to 17 in Table 1.

- Class V: the voters of this class are indifferent between the three candidates, thus they approve all the three candidates(type 19).

If we denote by $n_{t}$ the number of voter of type $t$, a voting situation is an 19-tuple $\tilde{n}=$ $\left(n_{1}, n_{2}, \ldots, n_{t}, \ldots, n_{19}\right)$ that indicates the total number $n_{t}$ of voters casting each type of preferences such that $\sum_{t=1}^{19} n_{t}=n$. We denote by $p_{t}$ the probability that a voter chooses the preference type $t$ such that $\sum_{t=1}^{19} p_{t}=1$. In Table $2, S(a)$ denotes the AV score candidate $a$ given the labels of Table 1.

Given $a, b \in A$, we denote by $n_{a b}$ the total number of voters who strictly prefer $a$ to $b$. If $n_{a b}>n_{b a}$, we say that $a$ majority dominates candidate $b$; or equivalently, $a$ beats $b$ in a pairwise

\footnotetext{
5 Under impartial anonymous culture-like assumptions, voting situations are assumed to be equally likely.

6 It is assumed that voters vote sincerely. So, we are not concerned with strategic behaviors.
} 
Table 1 The 19 possible preferences types with three candidates

\begin{tabular}{|c|c|c|c|c|c|}
\hline Class I: & $\begin{array}{l}\underline{a} \succ b \succ c \\
\underline{a} \succ c \succ b \\
\underline{b} \succ a \succ c \\
\underline{b} \succ c \succ a \\
\underline{c} \succ a \succ b \\
\underline{c} \succ b \succ a\end{array}$ & $\begin{array}{l}p_{1} \\
p_{2} \\
p_{3} \\
p_{4} \\
p_{5} \\
p_{6}\end{array}$ & Class II: & $\begin{array}{l}\underline{a} \succ \underline{b} \succ c \\
\underline{a} \succ \underline{c} \succ b \\
\underline{b} \succ \underline{a} \succ c \\
\underline{b} \succ \underline{c} \succ a \\
\underline{c} \succ \underline{a} \succ b \\
\underline{c} \succ \underline{b} \succ a\end{array}$ & $\begin{array}{l}p_{7} \\
p_{8} \\
p_{9} \\
p_{10} \\
p_{11} \\
p_{12}\end{array}$ \\
\hline Class III: & $\begin{array}{l}\underline{a} \sim \underline{b} \succ c \\
\underline{a} \sim \underline{c} \succ b \\
\underline{b} \sim \underline{c} \succ a\end{array}$ & $\begin{array}{l}p_{13} \\
p_{14} \\
p_{15}\end{array}$ & Class IV: & $\begin{array}{l}\underline{a} \succ b \sim c \\
\underline{b} \succ a \sim c \\
\underline{c} \succ a \sim b\end{array}$ & $\begin{array}{l}p_{16} \\
p_{17} \\
p_{18}\end{array}$ \\
\hline Class V: & $\underline{a} \sim \underline{b} \sim \underline{c}$ & $p_{19}$ & & & \\
\hline
\end{tabular}

Table 2 The AV score of the candidates

\begin{tabular}{lcc}
\hline \hline$S(a)$ & $=$ & $n_{1}+n_{2}+n_{7}+n_{8}+n_{9}+n_{11}+n_{13}+n_{14}+n_{16}+n_{19}$ \\
$S(b)$ & $=$ & $n_{3}+n_{4}+n_{7}+n_{9}+n_{10}+n_{12}+n_{13}+n_{15}+n_{17}+n_{19}$ \\
$S(c)$ & $=$ & $n_{5}+n_{6}+n_{8}+n_{10}+n_{11}+n_{12}+n_{14}+n_{15}+n_{18}+n_{19}$ \\
\hline \hline
\end{tabular}

majority voting. In such a case, we will simply write $a \mathbf{M} b$. Candidate $a$ is said to be the Condorcet winner (resp. the Condorcet loser) if for all $b \in A \backslash\{a\}, a \mathbf{M} b$ (resp. $b \mathbf{M} a$ ). If for a given voting situation we get $a \mathbf{M} b, b \mathbf{M} c$ and $c \mathbf{M} a$, this describes a majority cycle.

\subsection{PAV, the Condorcet winner and the Condorcet loser}

By the definition of PAV, it is obvious that with three candidates, if there is a Condorcet winner who belongs to the subset of majority-approved candidates, she is always elected if Rule $2 \mathrm{i}$ applies while rule 2ii will never apply. So, Rule 1i and 1ii can fail to elect the Condorcet winner. It is also obvious that if there is a Condorcet loser in a three-candidate election, she cannot be elected under Rule 2i and 2ii; so, PAV may elect the Condorcet loser only when Rule 1i or 1ii apply.

In order to motivate the paper, let us take the following two voting profiles ${ }^{7}$ each with 9 voters $V_{i}(i=1 . .9)$ in order to illustrate that in three-candidate elections, PAV can fail to select the Condorcet winner when she exists (under Rules 1i, 1ii and 2i) and that it can select the Condorcet loser (under Rules 1i and 1ii).

\begin{tabular}{|c|c|c|}
\hline \multicolumn{3}{|c|}{ Profile 1} \\
\hline$V_{1}: \underline{a} \succ c \succ b$ & $V_{2}: \underline{a} \succ c \succ b$ & $V_{3}: \underline{b} \succ c \succ a$ \\
\hline$V_{4}: \underline{b} \succ c \succ a$ & $V_{5}: \underline{c} \succ a \succ b$ & $V_{6}: \underline{c} \succ a \succ b$ \\
\hline$V_{7}: \underline{b} \succ a \succ c$ & $V_{8}: \underline{c} \succ b \succ a$ & $V_{9}: \underline{a} \succ \underline{b} \succ c$ \\
\hline \multicolumn{3}{|c|}{ Profile 2} \\
\hline$\overline{V_{1}: \underline{a} \succ c \succ b}$ & $V_{2}: \underline{b} \succ a \succ c$ & $V_{3}: \underline{b} \succ c \succ a$ \\
\hline$V_{4}: b \succ c \succ a$ & $V_{5}: c \succ a \succ b$ & $V_{6}: c \succ a \succ b$ \\
\hline$V_{7}: \underline{c} \succ a \succ b$ & $V_{8}: \underline{a} \succ \underline{b} \succ c$ & $V_{9}: \underline{c} \succ \underline{b} \succ a$ \\
\hline
\end{tabular}

Under both profiles, the reader can check that $c$ is the Condorcet winner and $b$ is the Condorcet loser. Under the first profile, we get $S(a)=S(c)=3$ and $S(b)=4$; no candidate gets the majority of the approvals ( 5 votes), according to Rule $1 \mathrm{i}, b$ is the winner since she is the AV winner. Thus,

\footnotetext{
7 Other examples are provided in Brams (2008), Brams and Sanver (2009).
} 
PAV under Rule 1i fails to select the Condorcet winner but selects the Condorcet loser. Under the second profile, $b$ is the unique majority-approved candidate with 5 votes; Rule 1ii applies and $b$ is the PAV winner: PAV under Rule 1i fails to elect the Condorcet winner but can select the Condorcet loser.

To get a profile under which Rule $2 \mathrm{i}$ applies and that PAV fails to select the Condorcet winner, the reader only need to add the following groups of voters to Profile 1: 3 voters with $\underline{a} \succ \underline{b} \succ c, 2$ voters with $\underline{a} \succ c \succ b, 3$ voters with $\underline{c} \succ \underline{a} \succ b$ and 4 voters with $\underline{c} \succ \underline{b} \succ a$.

The profiles we just used illustrate that under some voting situations, PAV can fail to elect the Condorcet winner when she exists and that it can elect the Condorcet loser when she exists. These two behaviors of PAV are just rare oddities or are a common occurrence? The aim of this paper is then to provide an answer to this question. So, we compute the Condorcet efficiency of PAV and its probability of electing the Condorcet loser for voting situations with three candidates. Before starting this task, we need to define a probability model for this.

\subsection{The probability model: the Extended Impartial Culture assumption}

The Impartial Culture (IC) assumption, first introduced in the social choice literature by Gehrlein and Fishburn (1976), is one of the hypothesis used in the social choice literature when computing the likelihood of voting events. Under IC, it is assumed that each voter chooses her preference according to a uniform probability distribution. When only strict ranking are allowed with $m$ candidates, IC gives a probability $\frac{1}{m !}$ for each of the $m$ ! rankings to be chosen independently. The likelihood of a given voting situation $\tilde{n}=\left(n_{1}, n_{2}, \ldots, n_{t}, \ldots, n_{m !}\right)$ is

$$
\operatorname{Prob}\left(\tilde{n}=\left(n_{1}, n_{2}, \ldots, n_{t}, \ldots, n_{m !}\right)\right)=\frac{n !}{\prod_{t=1}^{m !} n_{t} !} \times(m !)^{-n}
$$

For more details about the IC assumption, see among others Berg and Lepelley (1994), Gehrlein and Fishburn (1980b, 1976), Gehrlein and Lepelley (2010, 2017). According to Gehrlein (1979), one can derive the likelihood of most voting events under the IC assumption by using existing results on the representations of quadrivariate normal rules as suggested by Plackett (1954). ${ }^{8}$ GehrleinFishburn's technique usually needs a good knowledge of the existing formulas in statistics for the representation of quadrivariate positive orthants (Abrahamson, 1964, David and Mallows, 1961, Gehrlein, 1979).

When indifference is allowed, the Impartial Weak Ordering Culture (IWOC) was introduced by Gehrlein and Fishburn (1980a) as an extension of IC. See Gehrlein and Lepelley (2015, 1998), Gehrlein and Valognes (2001), Lepelley and Martin (2001), Merlin and Valognes (2004) for a non exhaustive review of theoretical works taken under IWOC-like assumptions. Recently, Diss et al. (2010) provided an extension of IC that allows the possibility that voters could have dichotomous preferences with complete indifference between two of the candidates and also the possibility of a complete indifference between all three candidates: the Extended Impartial Culture (EIC) assumption. Let us describes how it works. Consider the 5 classes of preferences described in Table 1 and let us denote by $k_{1}$ the probability that a voter's preference belongs to Class I; by $k_{2}$ the probability that a voter's preference belongs to Class II; by $k_{3}$ the probability that a voter's preference belongs to Class III; by $k_{4}$ the probability that a voter's preference belongs to Class IV and by $k_{5}$ the probability that a voter's preference belongs to Class V such that $k_{1}+k_{2}+k_{3}+k_{4}+k_{5}=1$. Under EIC, it is assumed that the rankings within a class are equally likely: $p_{t}=\frac{k_{1}}{6}$ for $t=1,2, \ldots, 6, p_{t}=\frac{k_{2}}{6}$ for $t=7,8, \ldots, 12, p_{t}=\frac{k_{3}}{3}$ for $t=13,14,15, p_{t}=\frac{k_{4}}{3}$ for $t=16,17,18$ and $p_{19}=k_{5}$.

\footnotetext{
8 Assume $\left(X_{1}, X_{2}, \cdots, X_{n}\right)$ a vector of $n$ random variables with a nonsingular multivariate normal distribution. Plackett (1954) evaluated the probability $P\left(X_{1}>x_{1}, X_{2}>x_{2}, \cdots, X_{n}>x_{n}\right)$; he ended with a reduction formula of this probability based on the numerical quadrature for $n=3,4$.
} 
With the 19 preference types of Table 1, the likelihood of a given voting situation $\tilde{n}=$ $\left(n_{1}, n_{2}, \ldots, n_{t}, \ldots, n_{19}\right)$ is given by

$$
\operatorname{Prob}\left(\tilde{n}=\left(n_{1}, n_{2}, \ldots, n_{t}, \ldots, n_{19}\right)\right)=\frac{n !}{\prod_{t=1}^{19} n_{t} !} \times \prod_{t=1}^{19} p_{t}^{n_{t}}
$$

Diss et al. (2010) used EIC to analyze the Condorcet efficiency of AV and all the scoring rules. They also provided the limiting probability that a Condorcet winner exists as follows ${ }^{9}$ :

$$
P_{\text {Con }}^{\infty}=\frac{3}{4}+\frac{3}{2 \pi} \arcsin \left(\frac{k_{1}+k_{2}+k_{3}+k_{4}}{3 k_{1}+3 k_{2}+2 k_{3}+2 k_{4}}\right)
$$

Given that $k_{1}+k_{2}+k_{3}+k_{4}+k_{5}=1$, we can rewrite $P_{C o n}^{\infty}$ :

$$
\begin{aligned}
P_{C o n}^{\infty}\left(k_{34}, k_{5}\right) & =\frac{3}{4}+\frac{3}{2 \pi} \arcsin \left(\frac{1-k_{5}}{3-k_{3}-k_{4}-3 k_{5}}\right) \\
& =\frac{3}{4}+\frac{3}{2 \pi} \arcsin \left(\frac{1-k_{5}}{3-k_{34}-3 k_{5}}\right) \text { with } k_{34}=k_{3}+k_{4}
\end{aligned}
$$

\section{Probability that PAV elects the Condorcet winner}

By computing the Condorcet efficiency of PAV, we first turn to that of AV. Diss et al. (2010) compute the Condorcet efficiency of AV under EIC assumption by assuming that $p_{19}=0$. They made this assumption because the preference type of Class $\mathbf{V}$ has no impact on the outcome under $\mathrm{AV}$; this is not the case under PAV where type 19 can really matter. Gehrlein and Lepelley (2015) provide a representation of the limiting probability of the Condorcet efficiency of AV by assuming that $p_{19} \geq 0$. In this paper, we provide an alternative form of this representation, then we move to that of PAV.

Given the voting situation $\tilde{n}$ on $A=\{a, b, c\}$, assume that candidate $a$ is the Condorcet winner; this means that $a \mathbf{M} b$ and $a \mathbf{M} b$. Using the labels of Table 1 these are respectively equivalent to Equations 1 and 2 .

$$
\begin{aligned}
& n_{1}+n_{2}-n_{3}-n_{4}+n_{5}-n_{6}+n_{7}+n_{8}-n_{9}-n_{10}+n_{11}-n_{12}+n_{14}-n_{15}+n_{16}-n_{17}>0 \\
& n_{1}+n_{2}+n_{3}-n_{4}-n_{5}-n_{6}+n_{7}+n_{8}+n_{9}-n_{10}-n_{11}-n_{12}+n_{13}-n_{15}+n_{16}-n_{18}>0
\end{aligned}
$$

Candidate $a$ is also the AV winner means that $S(a)>S(b)$ and $S(a)>S(c)$ which are respectively equivalent to Equations 3 and 4.

$$
\begin{aligned}
& n_{1}+n_{2}-n_{3}-n_{4}+n_{8}-n_{10}+n_{11}-n_{12}+n_{14}-n_{15}+n_{16}-n_{17}>0 \\
& n_{1}+n_{2}-n_{5}-n_{6}+n_{7}+n_{9}-n_{10}-n_{12}+n_{13}-n_{15}+n_{16}-n_{18}>0
\end{aligned}
$$

So, a voting situation under which AV elects the Condorcet winner is fully described by Equations 1 to 4 . In order to get a representation of the Condorcet efficiency of AV, we follow the same technique as Gehrlein and Fishburn (1978a). So, considering each of Equations 1 to 4, we define the following four discrete variables:

$$
\begin{aligned}
X_{1}=1 & : p_{1}+p_{2}+p_{5}+p_{7}+p_{8}+p_{11}+p_{14}+p_{16} \\
-1 & : p_{3}-p_{4}+p_{6}+p_{9}+p_{10}+p_{12} p_{15}+p_{17} \\
0 & : p_{13}+p_{18}+p_{19} \\
X_{2}=1 & : p_{1}+p_{2}+p_{3}+p_{7}+p_{8}+p_{9}+p_{13}+p_{16} \\
-1 & : p_{4}+p_{5}+p_{6}+p_{10}+p_{11}+p_{12}+p_{15}+p_{18} \\
0 & : p_{14}+p_{17}+p_{19}
\end{aligned}
$$

9 Notice that $P_{C o n}^{\infty}$ is also the probability that a Condorcet loser exists. 


$$
\begin{aligned}
X_{3}=\begin{aligned}
1 & : p_{1}+p_{2}+p_{8}+p_{11}+p_{14}+p_{16} \\
-1 & : p_{3}+p_{4}+p_{10}+p_{12}+p_{15}+p_{17} \\
0 & : p_{5}+p_{6}+p_{7}+p_{9}+p_{13}+p_{18}+p_{19}
\end{aligned} \\
X_{4}=\begin{aligned}
1 & : p_{1}+p_{2}+p_{7}+p_{9}+p_{13}+p_{16} \\
-1 & : p_{5}+p_{6}+p_{10}+p_{12}+p_{15}+p_{18} \\
0 & : p_{3}+p_{4}+p_{8}+p_{11}+p_{14}+p_{17}+p_{19}
\end{aligned}
\end{aligned}
$$

where $p_{i}$ is the probability that a voter who is randomly selected from the electorate is associated with the $i^{\text {th }}$ ranking of Table $1 ; X_{1}>0$ indicates that $a$ is preferred to $b$ and $X_{1}<0$ indicates the reverse. Similarly, $X_{2}>0$ indicates that $a$ is preferred to $c . X_{3}$ and $X_{4}$ respectively represent $S(a)-S(b)$ and $S(a)-S(c)$. Equations 1 to 4 fully describe a situation under which AV elects the Condorcet winner when the average value $\bar{X}_{j}$ of each of the $X_{j}$ (for $j=1,2,3,4$ ) are positive. According the Gehrlein and Fishburn (1978a,b), the probability of such a situation is equal to the joint probability $\bar{X}_{1}>0, \bar{X}_{2}>0, \bar{X}_{3}>0$ and $\bar{X}_{4}>0$; when $n \rightarrow \infty$, it is equivalent to the quadrivariate normal positive orthant probability $\Phi\left(R_{4}\right)$ such that $\bar{X}_{j} \sqrt{n} \geq E\left(\bar{X}_{j} \sqrt{n}\right)$ and $R_{4}$ is a correlation matrix between the variables $X_{j}$. The expectation value of $X_{j}$ is $E\left(X_{j}\right)=0$, the variances $\left(V\left(X_{j}\right)=E\left(X_{j}^{2}\right)\right)$ and covariances $\left(\operatorname{Cov}\left(X_{j}, X_{k}\right)=E\left(X_{j} X_{k}\right)\right)$ are:

$$
\begin{aligned}
V\left(X_{1}\right) & =V\left(X_{2}\right)=\frac{3 k_{1}+3 k_{2}+2 k_{3}+2 k_{4}}{3}=\frac{3-3 k_{5}-k_{34}}{3} \\
V\left(X_{3}\right) & =V\left(X_{4}\right)=\frac{2\left(k_{1}+k_{2}+k_{3}+k_{4}\right)}{3}=\frac{2\left(1-k_{5}\right)}{3} \\
\operatorname{Cov}\left(X_{1}, X_{2}\right) & =\operatorname{Cov}\left(X_{1}, X_{4}\right)=\operatorname{Cov}\left(X_{2}, X_{3}\right)=\operatorname{Cov}\left(X_{3}, X_{4}\right)=\frac{1-k_{5}}{3} \\
\operatorname{Cov}\left(X_{1}, X_{3}\right) & =\operatorname{Cov}\left(X_{2}, X_{4}\right)=2 \operatorname{Cov}\left(X_{1}, X_{2}\right)
\end{aligned}
$$

We derive the correlation matrix $R_{4}$ where the components $r_{j k}$ are $r_{j k}=r_{k j}=\frac{\operatorname{Cov}\left(X_{j}, X_{k}\right)}{\sqrt{V\left(X_{j}\right) V\left(X_{k}\right)}}$ :

$$
R_{4}=\left(\begin{array}{ccc}
1 \frac{1-k_{5}}{3-3 k_{5}-k_{34}} & \sqrt{\frac{2\left(1-k_{5}\right)}{3-3 k_{5}-k_{34}}} & \sqrt{\frac{1-k_{5}}{2\left(3-3 k_{5}-k_{34}\right)}} \\
1 & \sqrt{\frac{1-k_{5}}{2\left(3-3 k_{5}-k_{34}\right)}} & \sqrt{\frac{2\left(1-k_{5}\right)}{3-3 k_{5}-k_{34}}} \\
& 1 & \frac{1}{2} \\
& & 1
\end{array}\right)
$$

Gehrlein (1979) has developed a general representation of the orthant probabilities for obtaining numerical values of $\Phi\left(R_{4}\right)$ as a function of a series of bounded integrals over a single variable. Given $r_{j k}$ the correlation terms in the matrix $R_{4}$, this general representation is defined as follows:

$$
\begin{aligned}
\Phi\left(R_{4}\right)= & f\left(r_{12}, r_{13}, r_{14}, r_{23}, r_{24}, r_{34}\right) \\
= & \frac{1}{16}+\frac{\arcsin \left(r_{12}\right)+\arcsin \left(r_{13}\right)+\arcsin \left(r_{23}\right)}{8 \pi} \\
& +\frac{r_{14}}{4 \pi^{2}}\left[\int_{0}^{1} \frac{\arccos \left(\frac{r_{24} r_{34} z^{2}-r_{13} r_{14} r_{24} z^{2}+r_{12} r_{13}+r_{14}^{2} r_{23} z^{2}-r_{12} r_{14} r_{34} z^{2}-r_{23}}{\sqrt{\left(1-r_{14}^{2} z^{2}-r_{13}^{2}-r_{34}^{2} z^{2}+2 r_{13} r_{14} r_{34} z^{2}\right)\left(1-r_{24}^{2} z^{2}-r_{12}^{2}-r_{14}^{2} z^{2}+2 r_{12} r_{14} r_{24} z^{2}\right)}}\right)}{\sqrt{1-r_{14}^{2} z^{2}}} d z\right] \\
& +\frac{r_{24}}{4 \pi^{2}}\left[\int_{0}^{1} \frac{\arccos \left(\frac{r_{14} r_{34} z^{2}-r_{14} r_{23} r_{24} z^{2}+r_{12} r_{23}+r_{24}^{2} r_{13} z^{2}-r_{12} r_{24} r_{34} z^{2}-r_{13}}{\sqrt{\left(1-r_{24}^{2} z^{2}-r_{23}^{2}-r_{34}^{2} z^{2}+2 r_{23} r_{24} r_{34} z^{2}\right)\left(1-r_{24}^{2} z^{2}-r_{12}^{2}-r_{14}^{2} z^{2}+2 r_{12} r_{14} r_{24} z^{2}\right)}}\right)}{\sqrt{1-r_{24}^{2} z^{2}}} d z\right] \\
& +\frac{r_{34}}{4 \pi^{2}}\left[\int_{0}^{1} \frac{\arccos \left(\frac{r_{14} r_{24} z^{2}-r_{14} r_{23} r_{34} z^{2}+r_{13} r_{23}+r_{34}^{2} r_{12} z^{2}-r_{13} r_{24} r_{34} z^{2}-r_{12}}{\sqrt{\left(1-r_{24}^{2} z^{2}-r_{23}^{2}-r_{34}^{2} z^{2}+2 r_{23} r_{24} r_{34} z^{2}\right)\left(1-r_{14}^{2} z^{2}-r_{13}^{2}-r_{34}^{2} z^{2}+2 r_{13} r_{14} r_{34} z^{2}\right)}}\right)}{\sqrt{1-r_{34}^{2} z^{2}}} d z\right]
\end{aligned}
$$


Let us define the following quantities:

$$
\begin{aligned}
N_{1}\left(k_{34}, k_{5}, z\right) & =\frac{1}{2}(z+1)(z-1)\left(1-k_{5}-k_{34}\right)\left(3-3 k_{5}-k_{34}\right)^{-\frac{3}{2}}\left(2\left(1-k_{5}\right)\right)^{\frac{1}{2}} \\
N_{2}\left(k_{34}, k_{5}, z\right) & =-\frac{1}{4}\left(\left(k_{34}+7 k_{5}-7\right) z^{2}-4 k_{34}-10 k_{5}+10\right)\left(3-3 k_{5}-k_{34}\right)^{-\frac{3}{2}}\left(2\left(1-k_{5}\right)\right)^{\frac{1}{2}} \\
D_{1}\left(k_{34}, k_{5}, z\right) & =\frac{1}{4}(z-2)(z+2)\left(1-k_{5}-k_{34}\right)\left(3 k_{5}+k_{34}-3\right)^{-1} \\
D_{2}\left(k_{34}, k_{5}, z\right) & =\frac{\left(16-32 k_{5}-12 k_{34}+16 k_{5}^{2}+12 k_{5} k_{34}+2 k_{34}^{2}+\left(22 k_{5}+5 k_{34}-5 k_{5} k_{34}-11 k_{5}^{2}-11\right) z^{2}\right)}{2\left(3 k_{5}+k_{34}-3\right)^{2}} \\
D_{3}\left(k_{34}, k_{5}, z\right) & =-\frac{1}{4}\left(\left(k_{34}+7 k_{5}-7\right) z^{2}-4 k_{34}-10 k_{5}+10\right)\left(3 k_{5}+k_{34}-3\right)^{-1} ; \\
\mu\left(k_{34}, k_{5}, z\right) & =1+z^{2}\left(1-k_{5}\right)\left(2\left(3 k_{5}+k_{34}-3\right)\right)^{-1} \\
\nu\left(k_{34}, k_{5}, z\right) & =1+2 z^{2}\left(1-k_{5}\right)\left(3 k_{5}+k_{34}-3\right)^{-1}
\end{aligned}
$$

Then we derive Theorem 1 which gives the representation of the limiting Condorcet efficiency of AV.

Theorem 1 With three candidates and an infinite number of voters, the Condorcet efficiency of $A V$ is given by:

$$
\begin{aligned}
& C E_{A V}^{\infty}\left(k_{34}, k_{5}\right)=3\left(\frac{\Phi\left(R_{4}\right)}{P_{C o n}^{\infty}}\right) \\
& =\left(3\left(\pi+2 \arccos \left(\frac{k_{5}-1}{3 k_{5}+k_{34}-3}\right)\right)\right)^{-1} \times\left\{\frac{3 \pi}{4}+\frac{3}{2} \arcsin \left(\frac{1-k_{5}}{3-3 k_{5}-k_{34}}\right)\right. \\
& +\frac{3}{2} \arcsin \left(\sqrt{\frac{2\left(1-k_{5}\right)}{3-3 k_{5}-k_{34}}}\right)+\frac{3}{2} \arcsin \left(\frac{1}{2} \sqrt{\frac{2\left(1-k_{5}\right)}{3-3 k_{5}-k_{34}}}\right) \\
& +\frac{3}{2 \pi} \sqrt{\frac{2\left(1-k_{5}\right)}{3-3 k_{5}-k_{34}}}\left(\int_{0}^{1} \frac{\arccos \left(\frac{N_{1}\left(k_{34}, k_{5}, z\right)}{\sqrt{D_{1}\left(k_{34}, k_{5}, z\right) \times D_{2}\left(k_{34}, k_{5}, z\right)}}\right)}{\sqrt{\mu\left(k_{34}, k_{5}, z\right)}} d z\right) \\
& +\frac{3}{\pi} \sqrt{\frac{2\left(1-k_{5}\right)}{3-3 k_{5}-k_{34}}}\left(\int_{0}^{1} \frac{\arccos \left(\frac{N_{2}\left(k_{34}, k_{5}, z\right)}{\sqrt{D_{2}\left(k_{34}, k_{5}, z\right) \times D_{3}\left(k_{34}, k_{5}, z\right)}}\right)}{\sqrt{\nu\left(k_{34}, k_{5}, z\right)}} d z\right) \\
& \left.+\frac{3}{4}\left(\int_{0}^{1} \frac{1}{\sqrt{1-\frac{z^{2}}{4}}} d z\right)\right\}
\end{aligned}
$$

In Table 3, we report some computed values of $C E_{\mathrm{AV}}^{\infty}\left(k_{34}, k_{5}\right)$. It comes out that given the proportion of voters of each of the types 3,4 and 5, AV always elects the Condorcet winner in more than $75 \%$ of the cases. In this table, one can notice that for a given value of one parameter, the probability tends to increase with the other parameter. Notice that for $k_{5}=0$, we recover the same figures as Diss et al. (2010); for $k_{34}=0$, we get the same figures obtained by Gehrlein and Lepelley (2015).

Let us turn to the Condorcet efficiency of PAV. Assume without loss of generality that $a$ is the PAV winner on $A=\{a, b, c\}$. By definition, PAV elects the Condorcet winner when she exists if one of the following cases holds:

- i) when no candidate receive a majority of approvals, the Condorcet winner is the AV winner;

- ii) the Condorcet winner is among the majority approved candidates. 
Table 3 Some values of the probabilities $C E_{\mathrm{AV}}^{\infty}\left(k_{34}, k_{5}\right)$

\begin{tabular}{|c|c|c|c|c|c|c|c|c|c|c|c|}
\hline $\begin{array}{l}k_{5} \rightarrow \\
k_{34} \downarrow\end{array}$ & 0 & 0.1 & 0.2 & 0.3 & 0.4 & 0.5 & 0.6 & 0.7 & 0.8 & 0.9 & 1 \\
\hline 0 & 0.75720 & 0.75720 & 0.75720 & 0.75720 & 0.75720 & 0.75723 & 0.75723 & 0.75720 & 0.75720 & 0.75720 & - \\
\hline 0.1 & 0.76626 & 0.76725 & 0.76860 & 0.77031 & 0.77271 & 0.77604 & 0.78132 & 0.79074 & 0.81249 & - & - \\
\hline 0.2 & 0.77604 & 0.77835 & 0.78135 & 0.78525 & 0.79074 & 0.79890 & 0.81249 & 0.84033 & - & - & - \\
\hline 0.3 & 0.78684 & 0.79074 & 0.79578 & 0.80265 & 0.81252 & 0.82827 & 0.85815 & - & - & - & - \\
\hline 0.4 & 0.79890 & 0.80469 & 0.81249 & 0.82353 & 0.84036 & 0.87078 & - & - & - & - & - \\
\hline 0.5 & 0.81252 & 0.82095 & 0.83262 & 0.85005 & 0.88044 & - & - & - & - & - & - \\
\hline 0.6 & 0.82824 & 0.84036 & 0.85812 & 0.88818 & - & - & - & - & - & - & - \\
\hline 0.7 & 0.84705 & 0.86490 & 0.89448 & - & - & - & - & - & - & - & - \\
\hline 0.8 & 0.87081 & 0.89979 & - & - & - & - & - & - & - & - & - \\
\hline 0.9 & 0.90435 & - & - & - & - & - & - & - & - & - & - \\
\hline 1 & 1 & - & - & - & - & - & - & - & - & - & - \\
\hline
\end{tabular}

These two cases are disjoints and they fully describe the situations under which PAV always selects the Condorcet winner when she exists. So, given the voting situation $\tilde{n}$ on $A=\{a, b, c\}$, we get

- Case 1: when no candidate receive a majority of approvals

$$
\left\{\begin{array} { l } 
{ a \mathbf { M } b } \\
{ a \mathbf { M } b } \\
{ S ( b ) < S ( a ) } \\
{ S ( c ) < S ( a ) } \\
{ S ( a ) < \frac { n } { 2 } } \\
{ S ( b ) < \frac { n } { 2 } } \\
{ S ( c ) < \frac { n } { 2 } }
\end{array} \Rightarrow \left\{\begin{array} { l } 
{ a \mathbf { M } b } \\
{ a \mathbf { M } b } \\
{ S ( b ) < S ( a ) } \\
{ S ( c ) < S ( a ) } \\
{ S ( a ) < \frac { n } { 2 } }
\end{array} \Rightarrow \left\{\begin{array}{l}
n_{a b}-n_{b a}>0 \\
n_{a c}-n_{c a}>0 \\
S(a)-S(b)>0 \\
S(a)-S(c)>0 \\
-2 S(a)+n>0
\end{array}\right.\right.\right.
$$

The first four inequalities of the final system respectively correspond to Equations 1 to 4 . We derive the last inequality as follows:

$-n_{1}-n_{2}+n_{3}+n_{4}+n_{5}+n_{6}-n_{7}-n_{8}-n_{9}+n_{10}-n_{11}+n_{12}-n_{13}-n_{14}+n_{15}-n_{16}+n_{17}+n_{18}-n_{19}>0$

So, a voting situation under which no candidate is majority-approved and that PAV elects the Condorcet winner is fully described by Equations 1 to 5. We proceed as in the proof of Theorem 1 by defining for each equation, a discrete variable. As we have already defined the discrete variables $X_{1}, X_{2}, X_{3}$ and $X_{4}$ for Equations 1 to 4 , it remains for us to define the discrete variable $X_{5}$ associated with Equation 5 .

$$
\begin{aligned}
X_{5}= & 1: p_{3}+p_{4}+p_{5}+p_{6}+p_{10}+p_{12}+p_{15}+p_{17}+p_{18} \\
& -1: p_{1}+p_{2}+p_{7}+p_{8}+p_{9}+p_{11}+p_{13}+p_{14}+p_{16}+p_{19}
\end{aligned}
$$

Following Gehrlein and Fishburn (1978a,b), the probability of such a situation is equal to the joint probability $\bar{X}_{1}>0, \bar{X}_{2}>0, \bar{X}_{3}>0, \bar{X}_{4}>0$ and $\bar{X}_{5}>0$; when $n \rightarrow \infty$, it is equivalent to the quadrivariate normal positive orthant probability $\Phi\left(R_{5}\right)$ such that $\bar{X}_{j} \sqrt{n} \geq E\left(\bar{X}_{j} \sqrt{n}\right)$ and $R_{5}$ is a correlation matrix between the variables $X_{j}$. It remains for us to compute the following variances and covariances:

$$
\begin{aligned}
V\left(X_{5}\right) & =1 \\
\operatorname{Cov}\left(X_{1}, X_{5}\right) & =\operatorname{Cov}\left(X_{2}, X_{5}\right)=\operatorname{Cov}\left(X_{3}, X_{5}\right)=\operatorname{Cov}\left(X_{4}, X_{5}\right)=-2 \operatorname{Cov}\left(X_{1}, X_{2}\right)
\end{aligned}
$$


We derive the correlation matrix $R_{5}$ :

$$
R_{5}=\left(\begin{array}{cccc}
1 \frac{1-k_{5}}{3-3 k_{5}-k_{34}}-\frac{2 \sqrt{3}}{3}\left(\frac{\left(1-k_{5}\right) \sqrt{3-3 k_{5}-k_{34}}}{3-3 k_{5}-k_{34}}\right) & \sqrt{\frac{2\left(1-k_{5}\right)}{3-3 k_{5}-k_{34}}} & \sqrt{\frac{1-k_{5}}{2\left(3-3 k_{5}-k_{34}\right)}} \\
1 & -\frac{2 \sqrt{3}}{3}\left(\frac{\left(1-k_{5}\right) \sqrt{3-3 k_{5}-k_{34}}}{3-3 k_{5}-k_{34}}\right) & \sqrt{\frac{1-k_{5}}{2\left(3-3 k_{5}-k_{34}\right)}} & \sqrt{\frac{2\left(1-k_{5}\right)}{3-3 k_{5}-k_{34}}} \\
& 1 & -\sqrt{\frac{2\left(1-k_{5}\right)}{3}} & -\sqrt{\frac{2\left(1-k_{5}\right)}{3}} \\
& & 1 & \frac{1}{2} \\
& & & 1
\end{array}\right)
$$

Based on the Boole's Theorem, Gehrlein $(2017,2014)$ developed a general representation of the orthant probabilities for obtaining numerical values of $\Phi\left(R_{5}\right)$ as a linear combination of $\Phi\left(R_{4}\right)$ values, where matrices $R_{4}$ are obtained from correlation terms in $R_{5}$.

$$
\begin{aligned}
\Phi\left(R_{5}\right)= & \frac{1}{2}\left(f\left(r_{12}, r_{13}, r_{14}, r_{23}, r_{24}, r_{34}\right)-f\left(r_{12}, r_{13},-r_{15}, r_{23},-r_{25},-r_{35}\right)+f\left(r_{12},-r_{14},-r_{15},-r_{24},-r_{25}, r_{45}\right)\right. \\
& \left.-f\left(-r_{13},-r_{14},-r_{15}, r_{34}, r_{35}, r_{45}\right)+f\left(r_{23}, r_{24}, r_{25}, r_{34}, r_{35}, r_{45}\right)\right)
\end{aligned}
$$

Then, the probability of electing the Condorcet winner in this case is equal to $\frac{3 \Phi\left(R_{5}\right)}{P_{C o n}^{\infty}}$. For sake of space, we do not report the expression of $\Phi\left(R_{5}\right)$ here; This will also be the case throughout the paper for all the orthant probabilities associated with $5 \times 5$-correlation matrix.

- Case 2: the Condorcet winner is among the majority approved candidates.

$$
\left\{\begin{array} { l } 
{ a \mathbf { M } b } \\
{ a \mathbf { M } b } \\
{ S ( a ) > \frac { n } { 2 } }
\end{array} \Rightarrow \left\{\begin{array}{l}
n_{a b}-n_{b a}>0 \\
n_{a c}-n_{c a}>0 \\
2 S(a)-n>0
\end{array}\right.\right.
$$

The first two inequalities of this system respectively correspond to Equations 1 and 2; the last inequality is derive from Equation 5 . We then derive $R_{3}$ the matrix associated with the final system we obtain

$$
R_{3}=\left(\begin{array}{cc}
1 \frac{1-k_{5}}{3-3 k_{5}-k_{34}} & \frac{2\left(1-k_{5}\right)}{\sqrt{3\left(3-3 k_{5}-k_{34}\right)}} \\
1 & \frac{2\left(1-k_{5}\right)}{\sqrt{3\left(3-3 k_{5}-k_{34}\right)}}
\end{array}\right)
$$

Following David and Mallows (1961), we derive $\Phi\left(R_{3}\right)$ the corresponding positive-orthant probability.

$$
\Phi\left(R_{3}\right)=\frac{1}{8}+\frac{1}{4 \pi}\left[\arcsin \left(\frac{1-k_{5}}{3-3 k_{5}-k_{34}}\right)+2 \arcsin \left(\frac{2\left(1-k_{5}\right)}{\sqrt{3\left(3-3 k_{5}-k_{34}\right)}}\right)\right]
$$

In this case, the probability of electing the Condorcet winner is equal to $\frac{3 \Phi\left(R_{3}\right)}{P_{C o n}^{\infty}}$.

From the above, we derive Theorem 2 which gives the representation of the limiting Condorcet efficiency of PAV.

Theorem 2 With three candidates and an infinite number of voters, the Condorcet efficiency of $P A V$ is given by:

$$
C E_{P A V}^{\infty}\left(k_{34}, k_{5}\right)=3\left(\frac{\Phi\left(R_{5}\right)+\Phi\left(R_{3}\right)}{P_{C o n}^{\infty}}\right)
$$

In Appendix, we provide an external link to the full formula of $C E_{\mathrm{PAV}}^{\infty}\left(k_{34}, k_{5}\right)$. In Table 4 we display some values of $C E_{\mathrm{PAV}}^{\infty}\left(k_{34}, k_{5}\right)$.

In Table 4 , we notice that given a value of $k_{5}$, the probability tends to increase with $k_{34}$ and vice versa; PAV always elects the Condorcet winner in more than $88 \%$ of the cases. A comparison between the figures of Table 4 to those of Table 3 clearly allows us to see the dominance order of PAV on AV in terms of Condorcet's efficiency: for all $k_{5}$ and $k_{34}$, PAV is more efficient than AV; moreover, the efficiency of PAV is, in all cases, at least $10 \%$ higher than that of AV. 
Table 4 Some values of the probabilities $C E_{\mathrm{PAV}}^{\infty}\left(k_{34}, k_{5}\right)$

\begin{tabular}{|c|c|c|c|c|c|c|c|c|c|c|c|}
\hline $\begin{array}{l}k_{5} \rightarrow \\
k_{34 \downarrow}\end{array}$ & 0 & 0.1 & 0.2 & 0.3 & 0.4 & 0.5 & 0.6 & 0.7 & 0.8 & 0.9 & 1 \\
\hline 0 & 0.90229 & 0.89834 & 0.89543 & 0.89309 & 0.89109 & 0.88931 & 0.88767 & 0.88608 & 0.88443 & 0.88256 & - \\
\hline 0.1 & 0.90815 & 0.90464 & 0.90233 & 0.90083 & 0.90001 & 0.89995 & 0.90098 & 0.90415 & 0.91341 & - & - \\
\hline 0.2 & 0.91436 & 0.91137 & 0.90981 & 0.90935 & 0.91004 & 0.91231 & 0.91737 & 0.92932 & - & - & - \\
\hline 0.3 & 0.92095 & 0.91859 & 0.91795 & 0.91884 & 0.9216 & 0.92736 & 0.93979 & - & - & - & - \\
\hline 0.4 & 0.92799 & 0.92642 & 0.92696 & 0.92968 & 0.9355 & 0.94759 & - & - & - & - & - \\
\hline 0.5 & 0.93557 & 0.93501 & 0.93717 & 0.94259 & 0.95391 & - & - & - & - & - & - \\
\hline 0.6 & 0.94381 & 0.94463 & 0.94916 & 0.95941 & - & - & - & - & - & - & - \\
\hline 0.7 & 0.95288 & 0.95576 & 0.96452 & - & - & - & - & - & - & - & - \\
\hline 0.8 & 0.96316 & 0.96967 & - & - & - & - & - & - & - & - & - \\
\hline 0.9 & 0.97555 & - & - & - & - & - & - & - & - & - & - \\
\hline 1 & 1 & - & - & - & - & - & - & - & - & - & - \\
\hline
\end{tabular}

\section{Probability that AV and PAV elect the Condorcet loser}

Lepelley (1993) showed under an extension of IC assumption that if preferences are single-peaked, the election of the Condorcet loser is much less frequent with AV than with the Plurality rule. More recently, Gehrlein et al. (2016) built a framework to compare AV and the Plurality rule and they found under impartial anonymous culture-like assumptions that AV is much less susceptible to elect the Condorcet loser than the Plurality rule. Notice that Gehrlein et al. (2016) investigated different scenarios on voters' preferences included the one assumed in this paper. In this section, we first reconsider the likelihood of AV to elect the Condorcet loser when she exists under the EIC assumption in three-candidate election.

Given the voting situation $\tilde{n}$ on $A=\{a, b, c\}$, assume that candidate $a$ is the Condorcet loser and she is the AV winner; this means that $b \mathbf{M} a, c \mathbf{M} a, S(a)>S(b)$ and $S(a)>S(c)$. Using the same technique as before, we derive the correlation matrix $\bar{R}_{4}$ as follows:

$$
\bar{R}_{4}=\left(\begin{array}{ccc}
1 \frac{x}{y}-\sqrt{\frac{2 x}{y}} & -\sqrt{\frac{x}{2 y}} \\
1-\sqrt{\frac{x}{2 y}} & -\sqrt{\frac{2 x}{y}} \\
1 & \frac{1}{2} \\
& 1
\end{array}\right)
$$

and following Gehrlein $(2017,1979)$, we derive Theorem 3. 
Theorem 3 With three candidates and an infinite number of voters, the probability that AV elects the Condorcet loser under the EIC assumption is given by

$$
\begin{aligned}
& C L_{A V}^{\infty}\left(k_{34}, k_{5}\right)=3\left(\frac{\Phi\left(\bar{R}_{4}\right)}{P_{C o n}^{\infty}}\right) \\
& =\left(3\left(\pi+2 \arccos \left(\frac{k_{5}-1}{3 k_{5}+k_{34}-3}\right)\right)\right)^{-1} \times\left\{\frac{3 \pi}{4}+\frac{3}{2} \arcsin \left(\frac{1-k_{5}}{3-3 k_{5}-k_{34}}\right)\right. \\
& +\frac{3}{2} \arcsin \left(\sqrt{\frac{2\left(1-k_{5}\right)}{3-3 k_{5}-k_{34}}}\right)+\frac{3}{2} \arcsin \left(\frac{1}{2} \sqrt{\frac{2\left(1-k_{5}\right)}{3-3 k_{5}-k_{34}}}\right) \\
& -\frac{3}{2 \pi} \sqrt{\frac{2\left(1-k_{5}\right)}{\left(3-3 k_{5}-k_{34}\right)}}\left(\int_{0}^{1} \frac{\arccos \left(\frac{N_{1}\left(k_{34}, k_{5}, z\right)}{\sqrt{D_{1}\left(k_{34}, k_{5}, z\right) \times D_{2}\left(k_{34}, k_{5}, z\right)}}\right)}{\sqrt{\mu\left(k_{34}, k_{5}, z\right)}} d z\right) \\
& -\frac{3}{\pi} \sqrt{\frac{2\left(1-k_{5}\right)}{3-3 k_{5}-k_{34}}}\left(\int_{0}^{1} \frac{\arccos \left(\frac{N_{2}\left(k_{34}, k_{5}, z\right)}{\sqrt{D_{2}\left(k_{34}, k_{5}, z\right) \times D_{3}\left(k_{34}, k_{5}, z\right)}}\right)}{\sqrt{\nu\left(k_{34}, k_{5}, z\right)}} d z\right) \\
& \left.+\frac{3}{4}\left(\int_{0}^{1} \frac{1}{\sqrt{1-\frac{z^{2}}{4}}} d z\right)\right\}
\end{aligned}
$$

\begin{tabular}{|c|c|c|c|c|c|c|c|c|c|c|c|}
\hline $\begin{array}{l}k_{5} \rightarrow \\
k_{34} \downarrow\end{array}$ & 0 & 0.1 & 0.2 & 0.3 & 0.4 & 0.5 & 0.6 & 0.7 & 0.8 & 0.9 & 1 \\
\hline 0 & 0.03709 & 0.03709 & 0.03709 & 0.03709 & 0.03709 & 0.03709 & 0.03709 & 0.03709 & 0.03709 & 0.03709 & - \\
\hline 0.1 & 0.03393 & 0.03355 & 0.03311 & 0.03253 & 0.03174 & 0.03063 & 0.02896 & 0.02609 & 0.02013 & - & - \\
\hline 0.2 & 0.03063 & 0.02989 & 0.02896 & 0.02775 & 0.02609 & 0.02374 & 0.02013 & 0.01379 & - & - & - \\
\hline 0.3 & 0.02724 & 0.02609 & 0.02464 & 0.02272 & 0.02013 & 0.01637 & 0.01051 & - & - & - & - \\
\hline 0.4 & 0.02374 & 0.02216 & 0.02013 & 0.01746 & 0.01379 & 0.00847 & - & - & - & - & - \\
\hline 0.5 & 0.02013 & 0.01805 & 0.01541 & 0.01193 & 0.0071 & - & - & - & - & - & - \\
\hline 0.6 & 0.01637 & 0.01379 & 0.01051 & 0.00612 & - & - & - & - & - & - & - \\
\hline 0.7 & 0.01249 & 0.00938 & 0.00538 & - & - & - & - & - & - & - & - \\
\hline 0.8 & 0.00847 & 0.00475 & - & - & - & - & - & - & - & - & - \\
\hline 0.9 & 0.00436 & - & - & - & - & - & - & - & - & - & - \\
\hline 1 & - & - & - & - & - & - & - & - & - & - & - \\
\hline
\end{tabular}

Table 5 reports some values of $C L_{\mathrm{AV}}^{\infty}\left(k_{34}, k_{5}\right)$. In this table, one can notice that given the value of one of the parameters, the probability tends to decrease with the other parameter. We notice that the probability is maximized $(3.709 \%)$ when there is no voters who are indifferent between their two preferred candidates $\left(k_{34}=0\right)$.

Table 5 Some values of the probabilities $C L_{\mathrm{AV}}^{\infty}\left(k_{34}, k_{5}\right)$

Let us now turn to the probability that PAV elects the Condorcet loser in order to envisage the comparison with AV. By definition, there are two cases under which the Condorcet loser may be elected when she exists. These two cases are disjoints and they fully describe the situations under which PAV selects the Condorcet loser when she exists. So, given the voting situation $\tilde{n}$ on $A=\{a, b, c\}$, by assuming that candidate $a$ is the Condorcet loser, we get

- Case 1: if no candidate is majority-approved 


$$
\left\{\begin{array} { l } 
{ b \mathbf { M } a } \\
{ c \mathbf { M } a } \\
{ S ( b ) < S ( a ) } \\
{ S ( c ) < S ( a ) } \\
{ S ( a ) < \frac { n } { 2 } } \\
{ S ( b ) < \frac { n } { 2 } } \\
{ S ( c ) < \frac { n } { 2 } }
\end{array} \Rightarrow \left\{\begin{array} { l } 
{ b \mathbf { M } a } \\
{ c \mathbf { M } a } \\
{ S ( b ) < S ( a ) } \\
{ S ( c ) < S ( a ) } \\
{ S ( a ) < \frac { n } { 2 } }
\end{array} \Rightarrow \left\{\begin{array}{l}
n_{b a}-n_{a b}>0 \\
n_{c a}-n_{a c}>0 \\
S(a)-S(b)>0 \\
S(a)-S(c)>0 \\
-2 S(a)+n>0
\end{array}\right.\right.\right.
$$

Following the same technique as before, we derive the correlation matrix $\widehat{R}_{5}$ :

$$
\widehat{R}_{5}=\left(\begin{array}{cccc}
1 \frac{1-k_{5}}{3-3 k_{5}-k_{34}} & -\sqrt{\frac{2\left(1-k_{5}\right)}{3-3 k_{5}-k_{34}}} & -\sqrt{\frac{1-k_{5}}{2\left(3-3 k_{5}-k_{34}\right)}} & \frac{2\left(1-k_{5}\right)}{\sqrt{3\left(3-3 k_{5}-k_{34}\right)}} \\
1 & -\sqrt{\frac{1-k_{5}}{2\left(3-3 k_{5}-k_{34}\right)}} & -\sqrt{\frac{2\left(1-k_{5}\right)}{3-3 k_{5}-k_{34}}} & \frac{2\left(1-k_{5}\right)}{\sqrt{3\left(3-3 k_{5}-k_{34}\right)}} \\
& 1 & \frac{1}{2} & -\frac{\sqrt{6\left(1-k_{5}\right)}}{3} \\
& & 1 & -\frac{\sqrt{6\left(1-k_{5}\right)}}{3} \\
& & & 1
\end{array}\right)
$$

- Case 2: if only the Condorcet loser receives a majority of approvals

$$
\left\{\begin{array} { l } 
{ b \mathbf { M } a } \\
{ c \mathbf { M } a } \\
{ S ( b ) < \frac { n } { 2 } } \\
{ S ( c ) < \frac { n } { 2 } } \\
{ S ( a ) > \frac { n } { 2 } }
\end{array} \Rightarrow \left\{\begin{array}{l}
n_{b a}-n_{a b}>0 \\
n_{c a}-n_{a c}>0 \\
2 S(a)-n>0 \\
-2 S(b)+n>0 \\
-2 S(c)+n>0
\end{array}\right.\right.
$$

Following the same technique as before, we derive the correlation matrix $\widetilde{R}_{5}$ :

$$
\widetilde{R}_{5}=\left(\begin{array}{cccc}
1 \frac{1-k_{5}}{3-3 k_{5}-k_{34}}-\frac{2\left(1-k_{5}\right)}{\sqrt{3\left(3-3 k_{5}-k_{34}\right)}} & 0 & -\frac{2\left(1-k_{5}\right)}{\sqrt{3\left(3-3 k_{5}-k_{34}\right)}} \\
1 & 0 & -\frac{2\left(1-k_{5}\right)}{\sqrt{3\left(3-3 k_{5}-k_{34}\right)}} & -\frac{2\left(1-k_{5}\right)}{\sqrt{3\left(3-3 k_{5}-k_{34}\right)}} \\
& 1 & \frac{-3+4 k_{5}}{3} & \frac{3-4 k_{5}}{3} \\
& & 1 & \frac{3-4 k_{5}}{3} \\
& & & 1
\end{array}\right)
$$

We then derive Theorem 4 which gives the representation of the limiting Condorcet efficiency of PAV.

Theorem 4 With three candidates and an infinite number of voters, the probability that PAV elects the Condorcet loser when she exists is given by:

$$
C L_{P A V}^{\infty}\left(k_{34}, k_{5}\right)=3\left(\frac{\Phi\left(\widehat{R}_{5}\right)+\Phi\left(\widetilde{R}_{5}\right)}{P_{\text {Con }}^{\infty}}\right)
$$

In Appendix, we provide an external link to the full formula of $C L_{\mathrm{PAV}}^{\infty}\left(k_{34}, k_{5}\right)$. Table 6 displays some values of $C L_{\mathrm{PAV}}^{\infty}\left(k_{34}, k_{5}\right)$ which teach us that PAV elects the Condorcet loser in less than $1.9 \%$. We also notice that given the value of one of the parameters, the probability tends to decrease with the other parameter. More, the probability tends to be maximized when there is no voters who are indifferent between their two preferred candidates $\left(k_{34}=0\right)$.

The comparison between the figures of Table 6 to those of Table 5 highlights at which extend PAV is less likely to elect the Condorcet loser than AV: the propensity of electing the Condorcet loser under $\mathrm{AV}$ is quite the double of that under PAV for all couple of values $\left(k_{5}, k_{34}\right)$. 
Table 6 Some values of the probabilities $C L_{\mathrm{PAV}}^{\infty}\left(k_{34}, k_{5}\right)$

\begin{tabular}{cccccccccccc}
\hline \hline $\begin{array}{c}k_{5} \rightarrow \\
k_{34 \downarrow} \downarrow\end{array}$ & 0 & 0.1 & 0.2 & 0.3 & 0.4 & 0.5 & 0.6 & 0.7 & 0.8 & 0.9 & 1 \\
\hline 0 & 0.01655 & 0.01701 & 0.01729 & 0.01750 & 0.01766 & 0.01780 & 0.01792 & 0.01804 & 0.01815 & 0.01828 & - \\
0.1 & 0.01515 & 0.01540 & 0.01545 & 0.01535 & 0.01513 & 0.01473 & 0.01402 & 0.01272 & 0.00988 & - & - \\
0.2 & 0.01370 & 0.01374 & 0.01354 & 0.01313 & 0.01246 & 0.01144 & 0.00977 & 0.00675 & - & - & - \\
0.3 & 0.01221 & 0.01203 & 0.01154 & 0.01078 & 0.00965 & 0.00791 & 0.00512 & - & - & - & - \\
0.4 & 0.01067 & 0.01024 & 0.00946 & 0.00832 & 0.00665 & 0.00413 & - & - & - & - & - \\
0.5 & 0.00907 & 0.00839 & 0.00729 & 0.00572 & 0.00345 & - & - & - & - & - \\
0.6 & 0.00742 & 0.00645 & 0.00500 & 0.00296 & - & - & - & - & - & - \\
0.7 & 0.00570 & 0.00442 & 0.00259 & - & - & - & - & - & - & - \\
0.8 & 0.00392 & 0.00229 & - & - & - & - & - & - & - & - \\
0.9 & 0.00202 & - & - & - & - & - & - & - & - & - \\
1 & - & - & - & - & - & - & - & - & - \\
\hline \hline
\end{tabular}

\section{Conclusion}

In this paper, we focused on the Preference Approval Voting (PAV) which is a voting rule combining approval and preferences. This rule was first introduced by Brams and Sanver (2009). As nothing is known on the propensity of this rule of electing the Condorcet winner/loser when she exists, the main objective of the paper was to fill this void. Under the extended impartial culture (EIC) assumption, we have provided for in three-candidate elections, representation of the limiting probability that PAV elects the Condorcet winner when she exists. In addition to the analysis of Diss et al. (2010) and Gehrlein and Lepelley (2015), we have provided another representation of the limiting Condorcet efficiency of Approval Voting (AV) under EIC. By definition, PAV is built to be more Condorcet-efficient than AV. Our analysis has helped us to highlight at which extend PAV performs better than AV on the Condorcet criterion. It comes out that AV always elects the Condorcet winner in more than $75 \%$ of the cases, while PAV does in more than $88 \%$ of the cases and that the efficiency of PAV is, in all cases, at least $10 \%$ higher than that of AV.

We also focused, in three-candidate elections, on the probability of electing the Condorcet loser when she exists. As part of our analysis, we have the representation of the limiting probability for $\mathrm{AV}$ and also for PAV. For both rules, it comes out that the probability is maximized when there is no voters who are indifferent between their two preferred candidates. By definition, we know that PAV is less susceptible to elect the Condorcet loser than AV in all the cases. We noted that in all the cases, the propensity of electing the Condorcet loser under AV is quite the double of that under PAV.

\section{Appendix}

For space constraints, here are the links to:

- The full representation of $C E_{\mathrm{PAV}}^{\infty}\left(k_{34}, k_{5}\right)$, the limiting probability that PAV elects the Condorcet winner when she exists.

https://www.dropbox.com/s/vfvgoyg8k391bnz/Full\%20CE_PAV.pdf?dl=0

- The full representation of $C L_{\mathrm{PAV}}^{\infty}\left(k_{34}, k_{5}\right)$, the limiting probability that PAV elects the Condorcet loser when she exists.

https://www.dropbox.com/s/k1og7q1pgbh16o6/Full\%20CL_PAV.pdf?dl=0 


\section{References}

Abrahamson I.G. (1964) Orthant Probabilities for the Quadrivariate Normal Distribution. The Annals of Mathematical Statistics 35(4): 1685-1703.

Berg S, Lepelley D. (1994) On probability models in voting theory. Statistica Neerlandica 48: 133-146.

Brams S. (2008) Mathematics and Democracy : Designing Better Voting and Fair-Division Procedures. Princeton University Press.

Brams S.J. and Fishburn P.C. (2007) Approval Voting. Springer-Verlag.

Brams S.J. and Fishburn P.C. (1978) Approval Voting. American Political Science Review 72 (3): $831-847$

Brams S.J., Fishburn P.C. and Merril S. III (1988a) The responsiveness of approval voting: Comments on Saari and van Newenhizen. Public Choice 59(2): 121-131.

Brams S.J., Fishburn P.C. and Merril S. III (1988b) Rejoinder to Saari and van Newenhizen. Public Choice 59(2): 149.

Brams S.J. and Sanver R (2009) Voting systems that combine approval and preferences. In: Brams SJ, Gehrlein WV, Roberts FS (eds) The mathematics of preference, choice and order. Springer, Berlin, pp 215-237.

David F.N and Mallows C.L (1961) The variance of Spearman's rho in normal samples. Biometrika 48:19-28.

Diss M., Merlin, V., and Valognes F. (2010) On the condorcet efficiency of approval voting and extended scoring rules for three alternatives. In: Laslier J-F, Sanver RM (eds) Handbook on approval voting. Springer, Berlin, pp 255-283.

Gehrlein W.V. (2017) Computing Multivariate Normal Positive Orthant Probabilities with 4 and 5 Variables. Technical report.

Gehrlein W.V. (2004) The Effectiveness of Weighted Scoring Rules when Pairwise Majority Rule Cycles Exist. Mathematical Social Sciences 47: 69-85.

Gehrlein W.V. (1979) A representation for quadrivariate normal positive orthant probabilities. Communications in Statistics 8: 349-358.

Gehrlein, W.V. and Fishburn P.C. (1980) The Paradox Of Voting: Effects of individual indifference and intransitivity. Journal of Public Economics 14: 83-94.

Gehrlein W.V., Fishburn P.C. (1980) Robustness of positional scoring over subsets of alternatives, Appl. Math. Optim. 6: 241-255.

Gehrlein W.V. and Fishburn P.C. (1976) The probability of the paradox of voting: A computable solution. Journal of Economic Theory, 13: 14-25.

Gehrlein W.V. and Fishburn P.C. (1978a) Coincidence probabilities for simple majority and positional voting rules. Soc Sci Res 7:272-283.

Gehrlein W.V. and Fishburn P.C. (1978b) Probabilities of election outcomes for large electorates. Journal of Economic Theory 19(1): 38-49.

Gehrlein W.V. and Lepelley D. (2015) The Condorcet Efficiency Advantage that Voter Indifference Gives to Approval Voting Over Some Other Voting Rules. Group Decis Negot 24:243-269

Gehrlein, W.V., Lepelley D. (2010) Voting paradoxes and group coherence. Springer.

Gehrlein, W.V., Lepelley, D. (2017) Elections, Voting Rules and Paradoxical Outcomes. Publisher: Springer-Verlag.

Gehrlein W.V. and Lepelley D. (1998) The Condorcet efficiency of approval voting and the probability of electing the Condorcet loser. Journal of Math Econ 29: 271-283.

Gehrlein W.V, Moyouwou I. and Lepelley D. (2016)A note on Approval Voting and electing the Condorcet loser. Mathematical Social Sciences 80:115-122.

Gehrlein W.V., Valognes F (2001) Condorcet efficiency: a preference for indifference. Social Choice Welf 18:193-205

Ju B-G (2010) Collective choices for simple preferences. In: Laslier J-F, Sanver MR (eds) Handbook on Approval Voting. Studies in Choice and Welfare, Springer-Verlag Berlin Heidelberg, pp 41-90.

Laslier J-F. and Sanver R.M (2010) Handbook on Approval Voting. Studies in Choice and Welfare. Springer Berlin Heidelberg. 
Lepelley D. (1993) On the probability of electing the Condorcet loser. Mathematical social Sciences 25: 105-116.

Lepelley D., Martin M. (2001) Condorcet's paradox for weak preference orderings. European Journal of Political Economy 17: 163-177.

Merlin, V., Valognes, F. (2004) The impact of indifferent voters on the likelihood of some voting paradoxes. Mathematical Social Sciences 48: 343-361.

Plackett R.L. (1954). A Reduction Formula for Normal Multivariate Integrals. Biometrika, 41 (3/4), 351-360.

Saari D. G. and van Newenhizen J. (1988a) The problem of indeterminancy in approval, multiple and truncated voting systems. Public Choice 59(2):101-120

Saari D. G. and van Newenhizen J. (1988b) Is approval voting an unmitigated evil? A response to Brams, Fishburn and Merril. Public Choice 59(2): 133-147.

$\mathrm{Xu}$ Y. (2010) Axiomatizations of approval voting. In: Laslier J-F, Sanver MR (eds) Handbook on Approval Voting. Studies in Choice and Welfare, Springer-Verlag Berlin Heidelberg, pp 91-102 